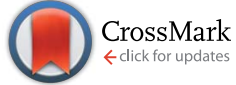

Cite this: J. Anal. At. Spectrom., 2015, 30,1080

Received 4th September 2014 Accepted 5th January 2015

DOI: 10.1039/c4ja00303a

www.rsc.org/jaas

\section{XAFS spectroscopy by an X-ray tube based spectrometer using a novel type of HOPG mosaic crystal and optimized image processing}

\author{
C. Schlesiger, ${ }^{\star a}$ L. Anklamm, $\dagger^{a}$ H. Stiel, ${ }^{b}$ W. Malzer ${ }^{a}$ and B. Kanngießer ${ }^{a}$
}

This paper presents recent achievements in laboratory based instrumentation for X-ray Absorption Fine Structure Spectroscopy (XAFS). The key component of the spectrometer is a HAPG mosaic crystal, which is employed in the von Hamos geometry. Due to the high efficiency of HAPG a low power micro focus $\mathrm{X}$-ray tube can serve as an X-ray source. Besides a description of the spectrometer, the paper covers the treatment of the CCD images in detail. The latter is crucial in order to entirely exploit the potential of the HAPG (Highly Annealed Pyrolitic Graphite) spectrometer. One section is dedicated to applications. As a first kind of application, the concentrations of two different iron species in mixtures are determined. A second kind of typical usage of XAFS is the determination of bond lengths from the EXAFS. This XAFS application is demonstrated with metallic $\mathrm{Ni}$ as a reference material.

\section{Introduction}

The spectroscopy of X-ray absorption fine structure (XAFS) is a well established and versatile method for the investigation of the electronic structure or for the identification of chemical compounds as well as bonding lengths and coordination within molecules. Synchrotron radiation facilities usually offer experimental stations for these X-ray absorption near edge structure (XANES) or extended X-ray absorption fine structure (EXAFS) methods. Both differ in the offered energy range around the selected ionization edge, where XANES is mainly in an interval of $50 \mathrm{eV}$ to $100 \mathrm{eV}$ around the edge and EXAFS describes the wider range. XANES and EXAFS are applied in many fields of research, like medicine, biology, geology, or material science.

Consequently, this wide range of applications triggered the interest in X-ray tube based spectrometers suitable for absorption spectroscopy. The major challenge for the development of such a spectrometer is the low brilliance of X-ray tubes compared to synchrotron radiation sources. Therefore, besides the use of highly brilliant X-ray tubes, efficient optical schemes play an important role in these developments. Another difference to measurements at Synchrotron radiation beamlines is that with this spectrometer there is no monochromatic excitation but the spectral resolving power is done in the detection channel and so we get one spectrum at once.

\footnotetext{
${ }^{a}$ Technical University Berlin, Sekr. EW 3-1, Hardenbergstr. 36, 10623 Berlin, Germany. E-mail: Christopher.Schlesiger@tu-berlin.de; Fax: +49 30314 23018; Tel: +49 30314 23015

${ }^{b}$ Max-Born-Institut, Max-Born-Straße 2a, 12489 Berlin, Germany

$\dagger$ Current affiliation: IAP e.V., Rudower Chaussee 29/31, 12489 Berlin, Germany.
}

Szlachetko et al. ${ }^{\mathbf{1}}$ recently presented a high-resolution transmission-type curved crystal spectrometer based on the modified DuMond slit geometry with the possibility to measure XAFS spectra. In the first order of reflection the spectrometer reached a spectral resolving power of $\Delta E$ ranging from $2.40 \mathrm{eV}$ up to $3.11 \mathrm{eV}$ for different slit widths.

Another spectrometer using a Rowland-circle monochromator and a low power X-ray tube was built up and presented by Seidler et al. ${ }^{2}$ This spectrometer has a spectral resolving power of $\Delta E=1 \mathrm{eV}$ in the range from $5 \mathrm{keV}$ up to 10 $\mathrm{keV}$, providing count rates for XAS comparable to those achieved at monochromatized spectroscopy beamlines at synchrotron radiation sources.

Wei et $a l .{ }^{3}$ demonstrated the use of doubly curved crystal optics in combination with a low power micro focus X-ray tube for XANES spectroscopy. Although the geometry is not designed for energy scanning, the authors could show that within a small energy range of around $100 \mathrm{eV}$, the spectral resolving power is sufficient for XANES. They further could show that the large solid angle of the optic facilitates reasonable acquisition times of about $1 \mathrm{~h}$ for an energy range of approximately $70 \mathrm{eV}$ at the Mn K absorption edge although a low power X-ray tube is used.

Several experiments for XAS in the laboratory were performed by Maeda et al., ${ }^{4}$ Sano et al., ${ }^{5}$ Nomura et al. ${ }^{6}$ and Matsushita et $a .^{7}$ The achieved spectral resolving power as well as the efficiency with the presented HAPG spectrometer is higher in comparison to the spectrometer used in these publications. Therefore especially the measurement time is reduced to get the desired statistical accuracy of better than $0.16 \%$. In contrast they focus the radiation onto the sample and therefore the influence of the lateral homogeneity is minimized. 
In this paper, we present recent progress in the development of a von Hamos spectrometer for XANES spectroscopy in transmission mode. This development was driven by the need for a versatile laboratory spectrometer which is capable of combining sufficient spectral resolving power with high efficiency. The key component of this setup is the X-ray optic, which is based on Highly Annealed Pyrolitic Graphite (HAPG) and combines sufficient spectral resolving power with high efficiency. With this HAPG X-ray optic XANES or EXAFS spectra can be acquired within minutes to hours, whereby an air cooled low power X-ray tube serves as an X-ray source. The possibilities and limitations with this spectrometer have to be considered especially keeping in mind the needed spectral resolving power for the XANES region and the high signal-to-noise ratio for EXAFS measurements.

HAPG is a recently developed mosaic crystal and very similar to Highly Oriented Pyrolitic Graphite (HOPG). Therefore, it also owns an integral reflectivity more than one order of magnitude higher than those of perfect crystals. Already HOPG was used for high resolution spectroscopy. Ice and Sparks ${ }^{8}$ were the first to evaluate the properties of a HOPG based von Hamos spectrometer. Legall et al. ${ }^{9}$ demonstrated the use of HAPG in a von Hamos spectrometer and its suitability for X-ray absorption spectroscopy.

In this paper, we will describe the characteristics and demonstrate the performance of such spectrometers. We exemplify the range of possible applications by two demonstration experiments. In a first experiment, the concentration ratio of two different iron compounds is determined by absorption spectroscopy using the spectral range near the edge, commonly known as XANES. The second application, which also is a common use of EXAFS at synchrotron radiation facilities, is the determination of bond lengths in molecules. This technique uses a wider spectral range to get the bonding distances. Both techniques can be used in applications for example like catalysis where it is necessary to know the chemical species by means of oxidation states and coordination within a molecule or mineralogy where the oxidation state and especially its ratio is of interest.

An entire section is dedicated to the evaluation of the X-ray image. The crystals mosaicity severely affects the X-ray image. In order to benefit from the high integral reflectivity it is crucial to evaluate the complete X-ray pattern. Furthermore, the mapping of the X-ray energies to the location of the CCD's pixels must be modeled properly in order to preserve the optimal spectral resolution. The image treatment, therefore, is a major step forward compared to the first demonstration experiments, where only the central row of pixels was used. ${ }^{9}$ The main advantage of the new image evaluation, presented in this contribution, is that most of the image's intensity is used without decreasing the spectral resolving power. Usually these images have been evaluated by using a rectangular integration of the image. This procedure ignores the image formation due to the chosen geometry and therefore must be adapted to ensure the highest spectral resolving power with the best statistics by using the maximal information given by the images.

\section{The HAPG von Hamos spectrometer}

The use of a cylindrically shaped crystal as a way to combine high spectral resolution with a high solid angle of detection was first published by von Hamos in $1934 .^{10}$ In this geometry, a cylindrically bent crystal is used as the dispersive element, which is hence flat in the dispersion plane. When the X-ray source is placed on the cylinder axis, all energies reflected on the crystal will sagittally be focused onto the cylinder axis again, hence increasing the solid angle in comparison to flat crystals for a given CCD camera size. Ice and Sparks ${ }^{8}$ were the first to use Highly Oriented Pyrolitic Graphite (HOPG) as the crystal in the von Hamos geometry, which has the advantage of exceptional high integral reflectivity, further enhancing the efficiency of this optical scheme. Fig. 1 shows the so-called mosaic focusing, the reason for the 20 to 50 times higher integral reflectivity of mosaic crystals. For further details, we refer the reader to the excellent publication of Ice and Sparks, ${ }^{8}$ who describe the properties of HOPG based von Hamos spectrometers in detail.

Highly Annealed Pyrolitic Graphite (HAPG) is a recently developed type of pyrolitic graphite with, compared to HOPG, lower mosaicity, which is particularly suited for applications requiring high spectral resolving power. It is a flexible material, with which strongly curved optics can be manufactured without corrupting the spectral resolution.

The X-ray sources we used for this work, were air cooled micro focus X-ray tubes with a power of $30 \mathrm{Watt}$, a spot size of 60 $\mu \mathrm{m}$ and molybdenum or copper as an anode material. An indirect X-ray CCD of the type Andor Newton A-DY940P-FO-CSI with a spatial resolution of $50 \mu \mathrm{m}$ and a size of $1 \times 0.25 \mathrm{inch}^{2}$ is used for the detection of the reflected X-rays. The HAPG optic has a size of $5 \times 5 \mathrm{~cm}^{2}$ and a bending radius of $150 \mathrm{~mm}$. The mosaicity of the HAPG film is $0.1^{\circ}$ and it has a thickness of 40 $\mu \mathrm{m}$. The large curvature radius results in large distances between the optical elements, e.g. more than half a meter for Xray energies around $8 \mathrm{keV}$. It is, however, required to minimize the degradation of the spectral resolving power by mosaicity effects as Ice and Sparks ${ }^{8}$ showed in their publication. Between the source and the optic and the optic and the detector respectively vacuum tubes are plugged in to decrease the absorption on air and hence the acquisition time. The specimen

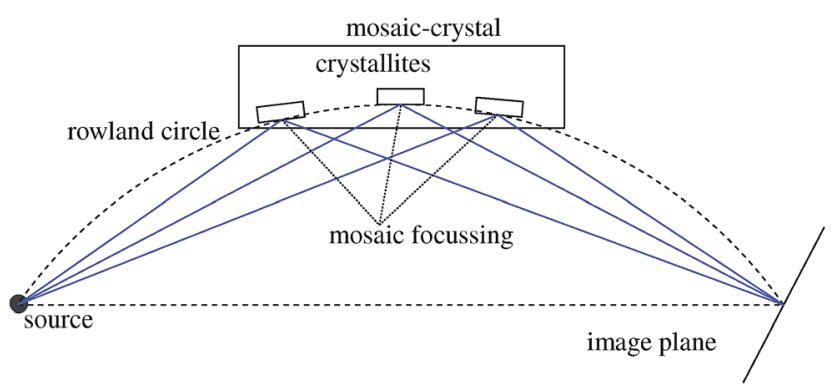

Fig. 1 The so-called mosaic focussing fulfills the requirements for the Johansson geometry for flat mosaic crystals. Due to the mosaic structure, there are crystallites appropriately oriented and positioned. 


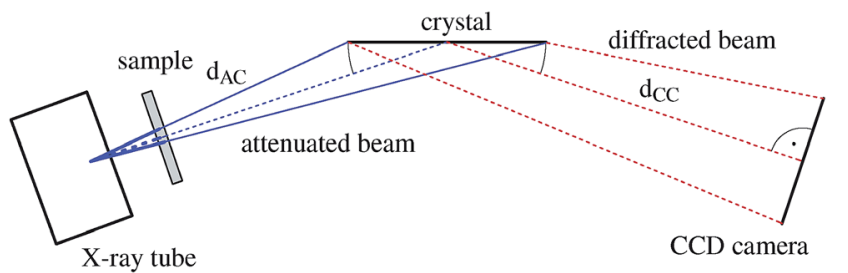

Fig. 2 The HAPG von Hamos spectrometer. From left to right: X-ray source, specimen in transmission mode, sagittally bent HAPG crystal as a dispersive element, CCD positioned perpendicular to the beam.

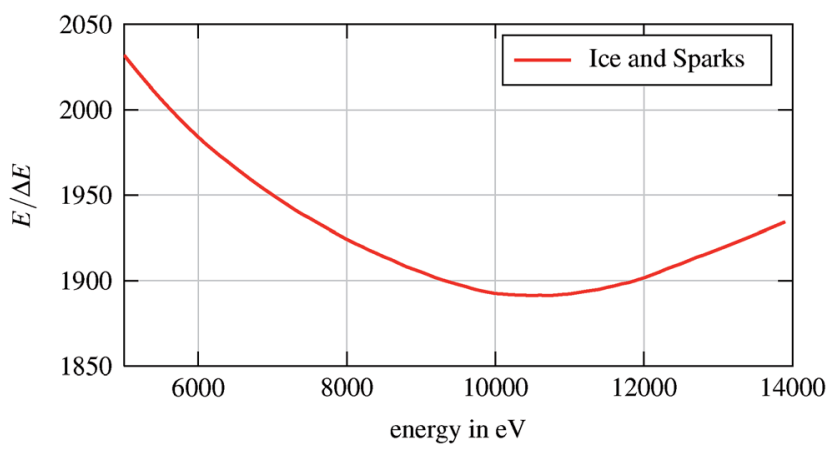

Fig. 3 The achievable spectral resolving power $E / \Delta E$ according to the calculations of Ice and Sparks ${ }^{8}$ for a curvature radius of $150 \mathrm{~mm}$, a crystal thickness of $40 \mu \mathrm{m}$, a mosaic spread of $0.1^{\circ}$ and a source size of $60 \mu \mathrm{m}$.

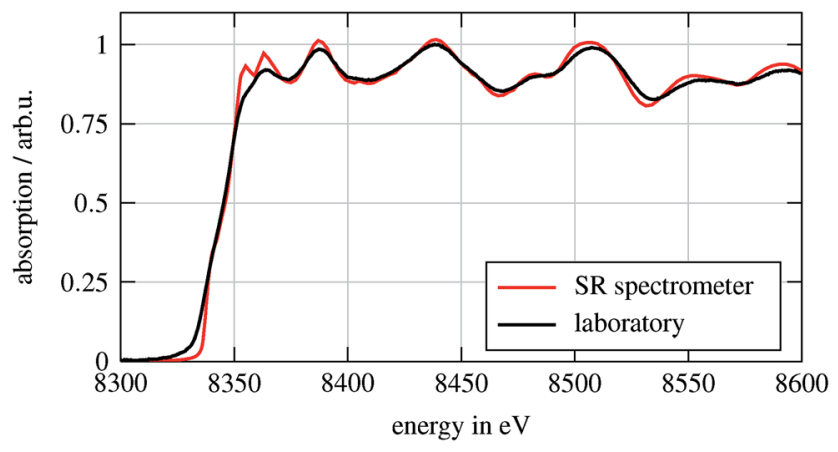

Fig. 4 The absorption spectrum of a $6 \mu \mathrm{m} \mathrm{Ni}$ foil is shown. The spectrum measured at a synchrotron radiation source has a better statistics, but nevertheless, almost all spectral features are distinguishable in the spectrum accumulated with the presented laboratory setup. Especially in the edge region are differences due to the lower spectral resolving power of the laboratory spectrometer which limit the use of XAS with this spectrometer by the means of evaluating XANES spectra.

is placed close to the exit window of the X-ray tube. A specimen size of a few $\mathrm{mm}^{2}$ is sufficient. A beam shutter is required to avoid image blurring during the readout of the CCD. A scheme of the setup is shown in Fig. 2.

In contrast to the von Hamos' conventional layout, the detector is oriented perpendicular to the beam. The small pixel size of current X-ray CCDs favors this geometry, which avoids a
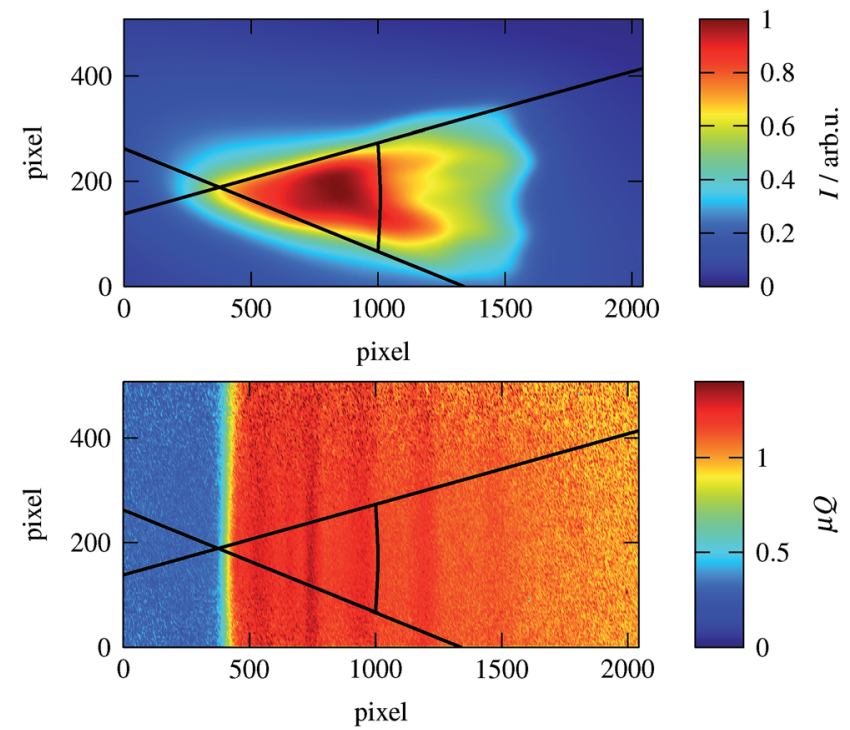

Fig. 5 Top: the CCD image without the sample (blank measurement). In this image all effects, for example crystal reflection, absorption along the air path and CCD efficiency are combined. Bottom: the CCD image of a measurement of Fe foil, already divided by the blank measurement, hence representing the spatial resolved absorption coefficient $\mu Q$. The black lines indicate the boundaries, where the intensity drops to half the value, and where a distinct pixel to energy conversion is given.

Table 1 The table compares the fraction of $\mathrm{Fe}$ in the mixtures with $\mathrm{Fe}_{2} \mathrm{O}_{3}$ determined by the reference method XRF with the fractions determined by XANES. The values are given in percent including the uncertainties as three times the standard deviation $(3 \sigma)$

Mass deposition fraction of pure iron/\%

\begin{tabular}{lll}
\hline XRF & XANES $(t=5 \mathrm{~min})$ & XANES $(t=30 \mathrm{~min})$ \\
\hline $90 \pm 1$ & $92 \pm 2$ & $90 \pm 1$ \\
$81 \pm 1$ & $84 \pm 3$ & $82 \pm 2$ \\
$70 \pm 1$ & $71 \pm 2$ & $70 \pm 1$ \\
$62 \pm 1$ & $61 \pm 2$ & $60 \pm 1$ \\
$54 \pm 1$ & $52 \pm 2$ & $52 \pm 1$ \\
$45 \pm 1$ & $44 \pm 1$ & $42 \pm 1$ \\
$32 \pm 1$ & $31 \pm 2$ & $31 \pm 1$
\end{tabular}

shallow angle of incidence and extends the range of energy, which can be detected with one image.

The spectral resolving power was determined by the examination of a characteristic X-ray tube line. We used the W L $\alpha 1$ line at $8.4 \mathrm{keV}$, which has a natural line width of $6.61 \mathrm{eV} .{ }^{11}$ The spectral broadening of the line by the spectrometer was determined with $4.6 \mathrm{eV}$. Fig. 3 displays the spectral resolving power of the HAPG spectrometer, according to the relations given by Ice and Sparks. ${ }^{8}$

With the X-ray tube based HAPG von Hamos spectrometer, XANES spectra can be taken from approximately $3.5 \mathrm{keV}$ up to $20 \mathrm{keV}$. The lower limit is given by geometrical restrictions whereas the higher limit is given by the detection limit of the CCD. In our case the useful range covers from $5 \mathrm{keV}$ up to $14 \mathrm{keV}$ 

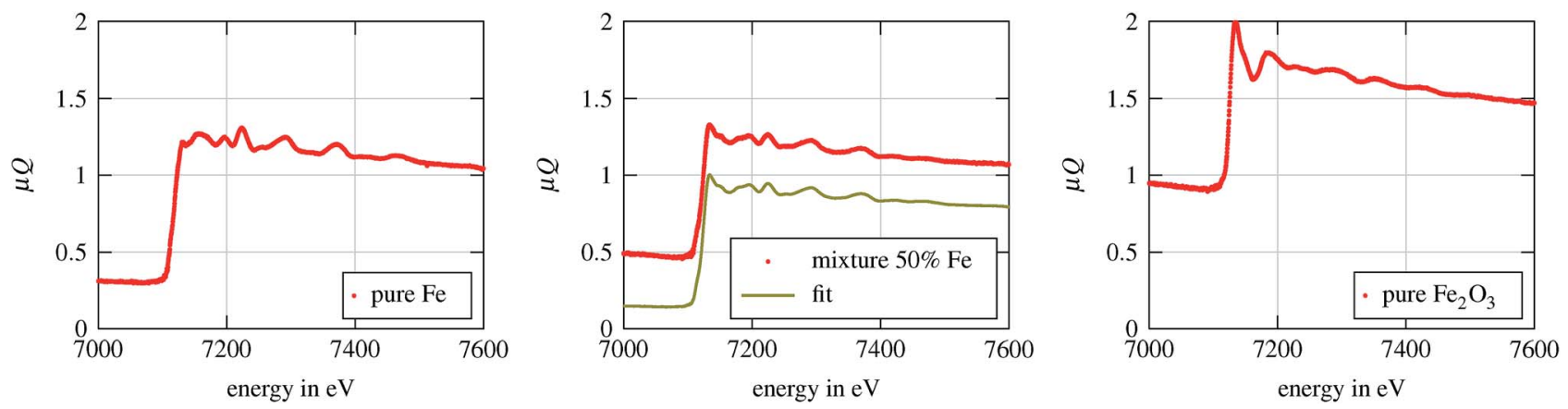

Fig. 6 From left to right are pictured the absolute absorption coefficients $\mu Q$ of the pure Fe, the mixture with $50 \%$ mass fraction of Fe and the pure $\mathrm{Fe}_{2} \mathrm{O}_{3}$. The offset and slope are due to scotch tape of each layer and are corrected within the evaluation of the mass ratio by measuring the pure scotch tape. The fit for the mixture is also shown in olive as a linear combination of the respective pure sample spectra. The fit is without the influence of scotch tape and therefore shifted to lower values for the absorption coefficient $\mu Q$.

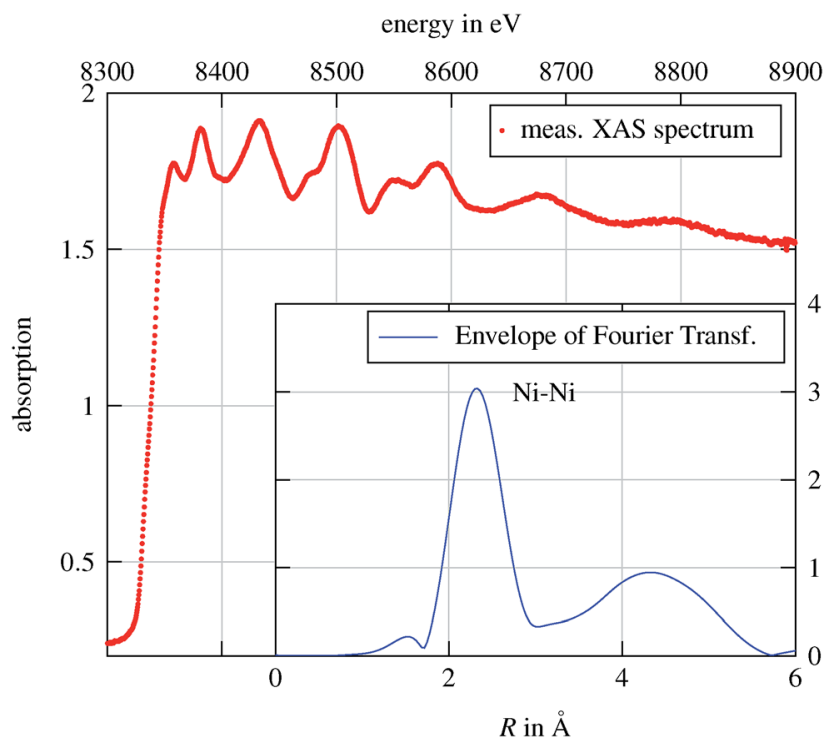

Fig. 7 The absorption spectrum of a Ni foil is shown. It consists of two separately measured spectra which are combined to provide good statistics in an energy interval of ca. $500 \mathrm{eV}$ starting at the absorption edge. The purpose is to get a good EXAFS signal for which statistics is rather more important than spectral resolving power. The inset shows the absolute of the Fourier transform of the EXAFS signal. The main peak is attributed to the $\mathrm{Ni}-\mathrm{Ni}$ distance for the first nearest neighbors. Additionally you can see the peak representing the $\mathrm{Ni}-\mathrm{Ni}$ distance for the second nearest neighbor which is significantly lower.

mainly limited by geometrical restrictions due to the lead shielding. K-edges can be analysed for elements from $\mathrm{Ti}$ to $\mathrm{Br}$, Ledges from Cs to $\mathrm{Hg}$ (whole L-edge series). Provided the sample thickness is within the optimal range between one and three times the absorption length at the respective absorption edge, acquiring a spectrum with reasonable signal to noise ratio requires $10 \mathrm{~min}$ to $30 \mathrm{~min}$. If the analyte's concentration is low, or if an excellent signal to noise ratio is required, the acquisition time will be higher.

Fig. 4 compares the XANES spectrum of a $6 \mu \mathrm{m}$ thick Ni foil obtained by our von Hamos spectrometer with a spectrum obtained at a synchrotron beamline. The latter was gathered at the X23A2 beamline at the National Synchrotron Light Source (NSLS), which covers an energy range from $4.9 \mathrm{keV}$ up to $30 \mathrm{keV}$ with a spectral resolving power of $E / \Delta E$ of 5000 at a bending magnet source. The effects caused by the lower spectral resolving power of the laboratory setup are visible in the broadening of the sharp spectral features close to the absorption edge. The excellent overall resemblance of the two spectra, however, proves the high quality the XAFS can be determined with. The HAPG based XANES spectrometer clearly is suited for the determination and discrimination of chemical compounds, even if they only show slight differences in their respective absorption fine structures.

\section{From image to spectrum}

In order to obtain high quality XANES spectra like displayed in Fig. 4, appropriate treatment of the image is crucial. The change in the geometry as well as the peculiarities of the mosaic crystal must be considered.

The geometry of the spectrometer presented differs from the classical von Hamos geometry. The surface of the CCD screen is aligned perpendicular to the central X-ray beam. Its surface, therefore intersects with the X-ray cones reflected from the crystal. The focusing condition is fulfilled for one energy only. The other energies are mapped to segments of ellipses. For shallow angles and the small cone segments of the XAFS spectrometer, the semi-major axis $r$ can be approximated by:

$$
r=c_{x} \pm \sqrt{\frac{\left(p x_{y}-c_{y}\right)^{2}}{\cos \theta_{\mathrm{B}}}+\left(p x_{x}-c_{x}\right)^{2}}
$$

with the focal position $\left(c_{x}, c_{y}\right)$, the Bragg angle $\theta_{\mathrm{B}}$ and the pixel coordinates $\left(p x_{x}, p x_{y}\right)$.

In Fig. 5 the boundary lines and an exemplary ellipse segment are drawn on top of an X-ray image. The image represents a part of the bremsstrahlung, whose intensity can be considered as being almost constant within the narrow energy band reflected by the crystal. Obviously the image of the reflection noticeably deviates from the geometrical shape 
predicted. These deviations are caused by the mosaicity of the HAPG crystal. In the next paragraphs, the three major effects will be discussed.

The reflectivity of the HAPG is not constant over the entire bandwidth. In Fig. 1, the mosaic focusing is shown. More precisely, it is shown for the central energy of the band width. For lower or higher energies, the number of crystallites contributing to the mosaic focusing decreases. Thus, the reflectivity of the crystal changes along the dispersion axis.

Also blurred are the edges along the boundary lines of the cone segment reflected by the cylinder segment. This blurring is another consequence of mosaicity. The crystallites are not only tilted in the meridional direction as shown in Fig. 1, but also perpendicular to the dispersion axis. This sagittal mosaic spread causes a broadening tangentially to the ellipses and therefore blurring of the edges.

In addition to these two image effects of mosaicity, HAPG thin films exhibit a considerable lateral inhomogeneity of their reflectivity. Reflection images of HAPG show brighter and darker regions.

For these reasons, the boundary lines of the cones and the focus point cannot be identified directly from the image. They are determined in two steps. First, the boundaries of individual columns of pixels are determined as the pixel, where the intensity dropped to half the value of the inner region of the cone segments. Arctangent functions are fitted to the edges. This works more reliably, if a few columns are summed up. The cone boundaries and the focus point then are determined by fitting two straight lines through the inflection points of these arctangent functions.

In summary, the image evaluation consists of the following steps. First, the dark current background is subtracted from the images and cosmic events at the CCD are removed. In the second step, the image taken with the analyte in the beam path is divided by the blank image, which is taken without an absorber or with an absorber, which resembles the specimens matrix, but does not contain the analyte.

This gives the additional advantage of directly and absolutely measuring the absorption coefficient $\mu Q$ given by the LambertBeer-law, with $\mu$ the mass attenuation coefficient, $Q$ the mass deposition of the analyte and $I$ and $I_{0}$ the two dimensional intensity distributions in the CCD planes with and without the sample, respectively,

$$
\mu Q=-\ln \left(\frac{I}{I_{0}}\right)
$$

for each of the CCD's pixels. This is due to the fact, that all effects, for example absorption on air and efficiency of the CCD, are included in both measurements with and without the sample. Hence the sample has the only effect on the attenuation given by eqn (2).

The two boundary lines which delimit the cones inner region are determined by the two step fitting procedure described above. The counts are integrated along the ellipses given by eqn (1). Finally the energy scale is calibrated by means of a reference spectrum, which defines the energy for one of the channels. The relation between the Bragg angle $\theta_{\mathrm{B}}$, defining the energy, and the pixel distance $\Delta r=r-r_{0}$ to the given reference point $r_{0}$ with the corresponding Bragg angle $\theta_{\mathrm{B}, 0}$ in the direction of the semimajor axis is given by: ${ }^{9}$

$$
\theta_{\mathrm{B}}=\theta_{\mathrm{B}, 0}-\arctan \left(\frac{\Delta r}{2 d}\right)
$$

with $d=D_{\mathrm{AC}}=d_{\mathrm{CC}}$ the component's distance from the source to the crystal as well as from the crystal to the CCD camera, respectively.

We like to conclude this section with a remark on the focus point. As only CCD pixels inside the inner region between the cone boundaries are used, the number of counts and, hence, the signal to noise ratio decreases. The XAFS spectrum will degrade in the vicinity of the focus point because there are only few pixels contributing. This is due to our improved image evaluation and the image formation which is due to the used spectrometer geometry. Therefore it is important to choose this focused energy, i.e. choosing the right components' distances, in an energy range with no spectral features. We usually focus an energy approximately $100 \mathrm{eV}$ before the absorption edge. This energy region is only used for normalization and does not have any oscillation, therefore good statistic is not necessary.

\section{Applications}

\subsection{Determination of concentration ratios}

A prominent application of XANES is the determination of the chemical species or of the mass fractions of chemical species in mixtures. In this section, we demonstrate the suitability of the laboratory spectrometer for the latter by using a linear combination of reference spectra of pure species. We investigated mixtures of $\mathrm{Fe}$ and of $\mathrm{Fe}_{2} \mathrm{O}_{3}$ powders in various concentration ratios.

A convenient way to prepare powder samples for XANES is the use of scotch tape, depositing a powder layer on its sticky side. We determined the mass deposition upon the individual pieces of scotch tape using X-ray fluorescence spectrometry. By stacking several pieces of loaded scotch tape, we could readily compose various mixtures and adapt the overall thickness of the specimen. Greatest care had to be taken for the homogeneity of the powder layers. In fact, the most prominent contribution to the uncertainties given in Table 1 for the reference value of the mass deposition originates from the inhomogeneity of the powder coverage.

For the XANES experiments, the spectrometer is equipped with a Mo X-ray tube, which was operated at $10 \mathrm{kV}$ high voltage to have no influence of higher reflection orders. The acquisition time for the reference spectrum was $30 \mathrm{~min}$. Spectra of the mixtures were taken within $5 \mathrm{~min}$ in a first run and within 30 min in a second one. Fig. 6 displays typical spectra for $30 \mathrm{~min}$ measurement time. Table 1 includes the results and the standard deviation for each run.

The mass ratios of $\mathrm{Fe}$ and $\mathrm{Fe}_{2} \mathrm{O}_{3}$ determined by XANES match the ratios determined by XRF within uncertainties. In this case, where the spectra of the mixture components are rather distinct and the thickness of the specimen can be adapted to the requirements of transmission XANES, the ratios 
can be determined with an uncertainty of around $3 \%$ within 5 min acquisition time and $2 \%$ within $30 \mathrm{~min}$, respectively.

\subsection{Determination of bond lengths}

Another important application of XAFS spectroscopy is its use for the study of the local structure. EXAFS is particularly suited to provide information on bond distances and coordination numbers.

EXAFS spectra typically have to cover a wider range of energy, up to $1 \mathrm{keV}$ above the absorption edge. Our XAFS spectrometer has a bandwidth with a full width of half maximum of $600 \mathrm{eV}$, however the bandwidth of optimal efficiency is only $400 \mathrm{eV}$. For the determination of bond length, therefore, we took two spectra with the center energy shifted by approximately $300 \mathrm{eV}$. The sum of these two spectra provides a sufficient signal to noise ratio up to an energy of $500 \mathrm{eV}$ above the absorption edge, corresponding to $c a$. $10 \AA^{-1}$ in $k$-space. The overall acquisition time was $3 \mathrm{~h}$ for measurements with and without the sample. Fig. 7 depicts the spectrum of metallic Ni taken. The inset shows the envelope of the Fourier transform indicating the bonding distances to the neighboring atoms to Ni. The major peak at 2.4 A comes from the Ni-Ni binding and agrees with the value of 2.5 A given by Sutton. ${ }^{12}$

\section{Conclusion and perspectives}

We presented a laboratory spectrometer for XANES and EXAFS measurements in transmission mode. The performance and the simplicity of the setup are mainly due to the use of the HAPG based von Hamos optic. A standard low power X-ray tube can serve as the X-ray source. A standard Peltier cooled X-ray CCD serves as the detector.

Although the spectral resolving power of around $E / \Delta E \approx$ 2000 is considerably lower than those achievable with the synchrotron based spectrometer, laboratory based XAFS can be used for identification of chemical elements, analysis of mixtures of compounds or for the determination of bonding lengths in molecules. Measurements, which could only be performed at synchrotron sources until now, can be carried out with such a laboratory spectrometer. Possible fields of application are catalysis, geology and others. The spectrometer scheme is also of interest for time resolved experiments as proposed in ref. 13 .

As the formation of the X-ray image is well understood and simulation and calculation tools are available, the spectrometer layout can be optimized for specific source properties and other requirements, or for EXAFS or XANES needs, by means of the imaged energy interval.

\section{Acknowledgements}

Inna Grigorieva and Alexander Antonov (Optigraph $\mathrm{GmbH}$ ) for providing HAPG optics and fruitful discussion on HAPG and HOPG. The Helmut Fischer GmbH for providing instrumentation for the setup.

\section{References}

1 M. Szlachetko, M. Berset, J.-C. Dousse, J. Hoszowska and J. Szlachetko, Rev. Sci. Instrum., 2013, 84, 093104.

2 G. Seidler, D. Mortensen, A. Remesnik, J. Pacold, N. Ball, N. Barry, M. Styczinski and O. Hoidn, Rev. Sci. Instrum., 2014, 85, 113906.

3 F. Wei, Z. W. Chen and W. M. Gibson, X-Ray Spectrom., 2009, 38, 382-385.

4 H. Maeda, H. Terauchi, K. Tanabe, N. Kamijo, M. Hida and H. Kawamura, Jpn. J. Appl. Phys., 1982, 21, 1342.

5 M. Sano, K. Taniguchi and H. Yamatera, Chem. Lett., 1980, 9, 1285-1286.

6 M. Nomura, K. Asakura, U. Kaminaga, T. Matsushita, K. Kohra and H. Kuroda, Bull. Chem. Soc. Jpn., 1982, 55, 3911-3914.

7 U. Kaminaga, T. Matsushita and K. Kohra, Jpn. J. Appl. Phys., 1981, 20, L355.

8 G. E. Ice and C. J. Sparks Jr., Nuclear Instruments and Methods in Physics Research Section A: Accelerators, Spectrometers, Detectors and Associated Equipment, 1990, vol. 291, pp. 110116.

9 H. Legall, H. Stiel, M. Schnürer, M. Pagels, B. Kanngießer, M. Müller, B. Beckhoff, I. Grigorieva, A. Antonov, V. Arkadiev and A. Bjeoumikhov, J. Appl. Crystallogr., 2009, 42, 572-579.

10 L. v. Hámos, Naturwissenschaften, 1932, 20, 705-706.

11 B. G. Gokhale, S. N. Shukla and R. N. Srivastava, Phys. Rev. A, 1983, 28, 858-862.

12 Table of interatomic distances and configuration in molecules and ions, ed. L. E. Sutton, Chemical Society, London, UK, Special publication No. 18 edn, 1965.

13 H. Stiel, M. Schnürer, H. Legall, W. Malzer, L. Anklamm, C. Schlesiger, K. A. Janulewicz, M. Iqbal and P. V. Nickles, Prospects of ultrafast X-ray absorption investigations using laboratory based sources, 2013, pp. $88490 \mathrm{H}$. 